\title{
PXO (Poly-XTAL Operations): MATLAB Codebase to Generate, Analyse and Export Complex 2D Spatio-Temporally Gradient Grain Structures
}

\author{
Sunil Anandatheertha ${ }^{1}$
}

\section{DOI: $10.21105 /$ joss. 03190}

\section{Software}

- Review ¿

- Repository ca

- Archive ca

Editor: Kevin M. Moerman ¿ Reviewers:

- Oyangbai90

- @ksyang2013

- Qlittlelazy6

Submitted: 07 February 2021 Published: 29 July 2021

\section{License}

Authors of papers retain copyright and release the work under a Creative Commons Attribution 4.0 International License (CC BY 4.0).
1 Coventry University, Priory street, Coventry, United Kingdom, CV1 5FB

\section{Statement of need}

Mathematicians, statistical mechanists and computational material scientists are interested in studying the spatiotemporal evolutionary aspects of, multi-classed partitioning of an ndimensional space. Four examples for this are given and the statement of need is provided in each.

The 1st example is from mathematics. Here, researchers are interested in the chaotic partitioning of an n-D bounded spatial domain and its spatiotemporal evolution under some governing rules (Mark Klien, 1999). A computational platform to study $n$-dimensional partitions of space is needed to enable this.

The 2nd example is from statistical mechanics. Here researchers are interested in importance sampling Monte-Carlo techniques studying the spatiotemporal evolution of the kinetics and thermodynamics of the distribution of multi-phase systems over some lattice. Though, exact solutions have been developed for the Ising model (Baxter, 1989), it is however, impractical for more complex models like the Q-state Pott's model, owing to the vastness of solution space. Here, a computational platform is needed to simulate such complex models which enables studying their complex behaviours.

The 3rd example is from fundamental computational materials science, where researchers are interested in the growth of grains (Weaire \& Mcmurry, 1996). Here the temporal evolution of spatial and thermodynamic parameters of multi-phase grain structures (Anderson et al., 1984), (Anderson et al., 1989) is studied at micro- and macro- scales. Though phase-field approaches are used, they are however, computational very intensive. Here, a computational platform is used to simulate grain growth in a simplified manner, and Monte-Carlo simulation techniques have been used to address this need (Anderson et al., 1984). A part of this research also touches upon understanding the kinematic and kinetic behaviour of grain structures in the presence of insoluble 2nd phase particles (Srolovitz et al., 1984) and how they interact with the grain boundaries. Some of these studies have tried to validate empirical models of grain growth (for parameter such as grain growth exponent), and empirical models pertaining to grain structure geometry such as the Zener equation (Manohar et al., 1998). As theparticle shape influences the Zener drag working against grain boundary evolution during grain growth (Li \& Easterling, 1990), and also that, nature presents irregularly shaped particles, computer models which can consider such particle shape and their spatial distribution, becomes essential in computer simulations of grain growth. In addition to these, it is also important to understand the grain growth kinetics. A software platform is needed where such studies can be made.

The 4th example is from applied computational materials science. Here, researchers use techniques such as crystal plasticity based finite element analysis to study a material's phase-partitioned thermo-mechanical response and texture evolution under applied thermomechanical loads (Roters et al., 2010). These are usually done at micro- and macro- scales. 
Some of such advanced analyses require parametric and realistically tessellated geometric morphologies of the constituent phases reflected in the virtual grain structures. Such virtual grain structures could either be realized using experimentally derived grain structures or by using purely computer generated grain structures. Such grain structures are generally used in crystal plasticity based finite element analysis (Tu et al., May 2021). Though Voronoi tessellated geometries of grain structures have been used before, they are simplifications and do not accurately represent the geometric irregularities presented by nature. However, it is difficult to generate parametric, spatially gradient and physically realistic grain structures with multiple temporally-low-gradient slices. It is also difficult for many, to acquire grain structures using experimental techniques like EBSD and even difficult to process them and use them numerically. Furthermore, such methods are also relevant to computational geology. A computer software program capable of preparing artificial and physically realistic grain structures for use in finite element analysis will be valuable.

\section{Summary}

Poly-XTAL operations (PXO) is a MATLAB based computational platform which can enable users to create and prepare realistic grain structures for finite element analysis, simulate complex grain growth phenomenon and achieve spatiotemporal partitioning of a 2D space into a set of closed regions. In addition to various in-built tools, PXO generated grain structures can be imported into 3rd party open source libraries such as MTEX (Hielscher \& Schaeben, 2008) and MTEX2Gmesh (Depriester \& Kubler, 2020) to tend to the specific requirements of the user. It allows easy inclusion of user defined physics based models for grain growth. Monte-Carlo simulations form the heart of PXO. However, Voronoi tessellation based grain structures can also be generated over different types of underlying lattices, with the added functionality of generating pixelated equivalents of Voronoi tessellation grain structures.

\section{Documentation}

All documentation, tutorials and test cases may be accessed from PXO/Wiki.

\section{State of the field}

Over the past few decades, MATLAB has emerged to be an easy-to-use, powerful and robust, high-level computational platform with ample documentation, and a fast growing scientific and engineering community. Poly-XTAL Operations, written in MATLAB, offers the relevant research community, an added advantage over other programming software, as MATLAB has a flat to moderate learning curve. Options like MCPM (Mason et al., 2015), NEPER (Quey, n.d.) and DREAM3D (Groeber \& Jackson, 2014) are available to model grain structures (Tu et al., May 2021). Though, these are powerful environments to deal with grain structure calculations, researchers from a non-software background may find it hard to use and/or modify them to their specific requirements. Implementing user developed solver algorithms in PXO is easy. PXO offers the added advantage of being able to be linked with MTEX (Hielscher \& Schaeben, 2008) to deal with crystallographic texture calculations and advanced grain structure analysis. As PXO offers tools to export as many available temporal slices of grain structures as the user wishes, to a format able to be used in ABAQUS software for finite element analysis (using MTEX2Gmesh (Depriester \& Kubler, 2020)), it offers a comparatively unique, viable and fast solution to researchers working in the field of Integrated Computational Materials Engineering (ICME) investigating structure-property-behaviour relationships. Additionally, PXO is able to combine this advantage with the ability to simulate grain growth in the presence of particles, 


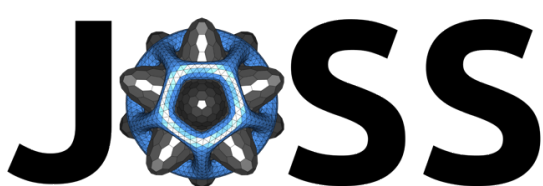

The Journal of Open Source Software

particle clusters, fibres, whiskers and temperature fields. Another unique advantage is the capability of generating morphologically complex grain structure distributions.

\section{Example grain structures}
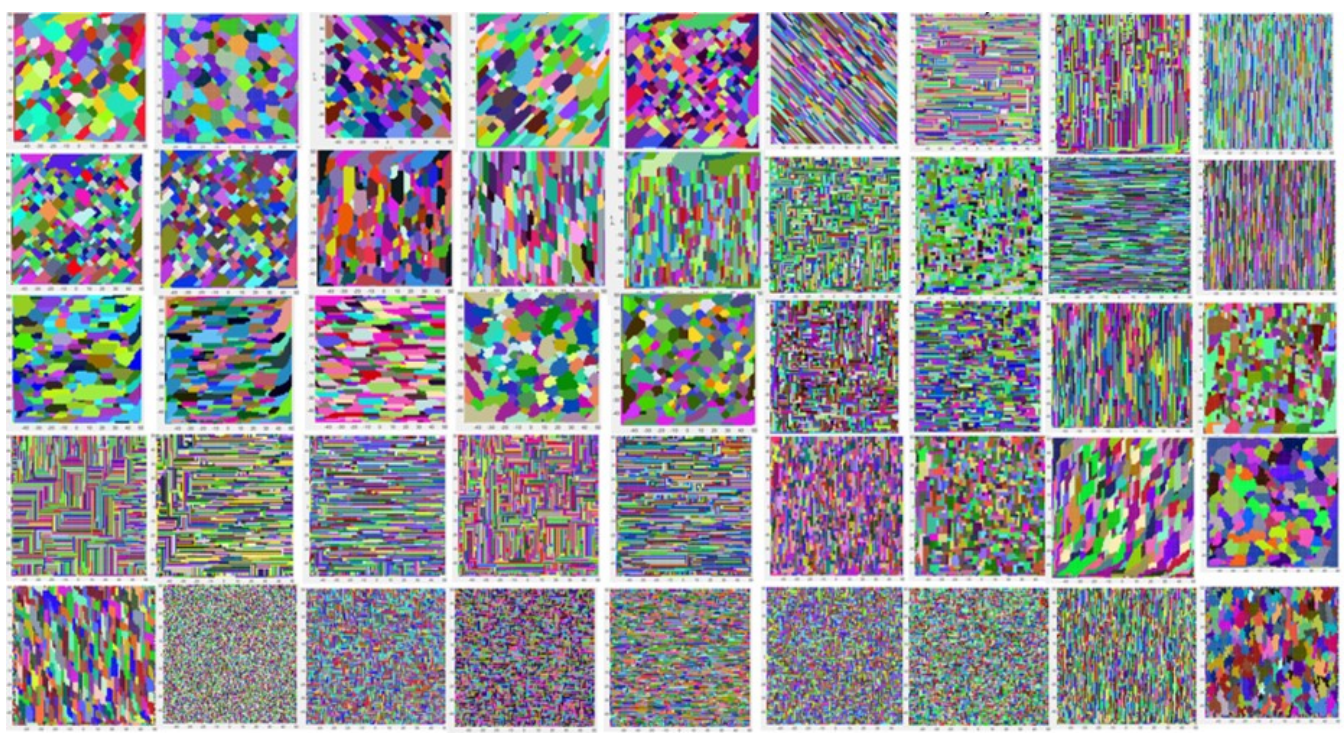

Figure 1: Example grain structures

The above figure shows some sample grain structures and space partitions, which can be generated using PXO. Tutorials have been provided to help the users to get started.

\section{Acknowledgements}

The author acknowledges the computational resources offered by the following institutions: Indian Institute of Science during 2013-2014, PES University during 2014-2017 and Coventry University during 2017-2021. The author also acknowledges Dr. Kishore T Kashyap (Department of Mechanical Engineering, PES University, Bengaluru, India, in 2010) for his theoretical inputs on grain growth, Dr. G Narayana Naik (Department of Aerospace Engineering, Indian Institute of Science, Bengaluru, India) and Dr. N G Subramania Udupa (Nagarjuna College of Engineering and Technology, Bengaluru, India) for supervising the author's master's project in 2012-2014.

\section{References}

Anderson, M. P., Grest, G. S., Doherty, R. D., Li, K., \& Srolovitz, D. J. (1989). Inhibition of grain growth by second phase particles: Three dimensional Monte Carlo computer simulations. Scripta Metallurgica, 23(5), 753-758. https://doi.org/10.1016/0036-9748(89) 90525-5

Anderson, M. P., Srolovitz, D. J., Grest, G. S., \& Sahni, P. S. (1984). Computer simulation of grain growth-I. Kinetics. Acta Metallurgica, 32(5), 783-791. https://doi.org/10.1016/ 0001-6160(84)90151-2

Anandatheertha, S., (2021). PXO (Poly-XTAL Operations): MATLAB Codebase to Generate, Analyse and Export Complex 2D Spatio- 3 Temporally Gradient Grain Structures. Journal of Open Source Software, 6(63), 3190. https://doi.org/10.21105/joss.03190 
Baxter, J., Rodney. (1989). Exactly Solved Model in Statistica. ISBN: 0120831805

Depriester, D., \& Kubler, R. (2020). MTEX2Gmsh: A tool for generating 2D meshes from EBSD data. Journal of Open Source Software, 5(52), 2094. https://doi.org/10.21105/ joss.02094

Groeber, M. A., \& Jackson, M. A. (2014). DREAM.3D: A Digital Representation Environment for the Analysis of Microstructure in 3D. Integrating Materials and Manufacturing Innovation, 3:5. https://doi.org/10.1186/2193-9772-3-5

Hielscher, R., \& Schaeben, H. (2008). A novel pole figure inversion method: specification of the MTEX algorithm. Journal of Applied Crystallography, 41.6, 1024-1037. https: //doi.org/10.1107/S0021889808030112

Li, W. B., \& Easterling, K. E. (1990). The influence of particle shape on zener drag. Acta Metallurgica Et Materialia, 38(6), 1045-1052. https://doi.org/10.1016/0956-7151(90) 90177-I

Manohar, P. A., Ferry, M., \& Chandra, T. (1998). Five Decades of the Zener Equation. ISIJ International, 38(9), 913-924. https://doi.org/10.2355/isijinternational.38.913

Mark Klien, J. (1999). Chapter 11: Polygon decomposition, Handbook of Computational Geometry (J.-R. Sack \& J. Urrita, Eds.; pp. 491-518). Elsevier Science B. V.

Mason, J. K., Lind, J., Li, S. F., Reed, B. W., \& Kumar, M. (2015). Kinetics and anisotropy of the Monte Carlo model of grain growth. Acta Materialia, 82, 155-166. https://doi. org/10.1016/j.actamat.2014.08.063

Quey, R. (n.d.). Neper: numerical descriptors of polycrystals (version 2.0), Available at http://neper.sourceforge.net/.

Roters, F., Eisenlohr, P., Hantcherli, L., Tjahjanto, D. D., Bieler, T. R., \& Raabe, D. (2010). Overview of constitutive laws, kinematics, homogenization and multiscale methods in crystal plasticity finite-element modeling: Theory, experiments, applications. Acta Materialia, 58(4), 1152-1211. https://doi.org/10.1016/j.actamat.2009.10.058

Srolovitz, D. J., Anderson, M. P., Grest, G. S., \& Sahni, P. S. (1984). Computer simulation of grain growth-III. Influence of a particle dispersion. Acta Metallurgica, 32(9), 1429-1438. https://doi.org/10.1016/0001-6160(84)90089-0

Tu, Y., Leen, S. B., \& Harrison, N. M. (May 2021). A high-fidelity crystal-plasticity finite element methodology for low-cycle fatigue using automatic electron backscatter diffraction scan conversion: Application to hot-rolled cobalt-chromium alloy. Proceedings of the Institution of Mechanical Engineers, Part L: Journal of Materials: Design and Applications. https://doi.org/10.1177/14644207211010836

Weaire, D., \& Mcmurry, S. (1996). Some Fundamentals of Grain Growth. Solid State Physics - Advances in Research and Applications, 50(C), 1-36. https://doi.org/10.1016/ S0081-1947(08)60603-7 